\title{
Low-dose ketamine does not improve the speed of recovery from depression in electroconvulsive therapy: a randomized controlled trial
}

\author{
Adrianna J. Woolsey, ${ }^{1 *}$ (iD Jalal A. Nanji, ${ }^{1 *}$ Chantal Moreau, ${ }^{2}$ Sudhakar Sivapalan, ${ }^{2}$ \\ Stephane L. Bourque, ${ }^{1}$ Alfonso Ceccherini-Nelli, ${ }^{2 \dagger}$ Ferrante S. Gragasin ${ }^{1 \dagger}$ \\ ${ }^{1}$ Department of Anesthesiology and Pain Medicine, University of Alberta, Edmonton, Alberta, Canada. ${ }^{2}$ Department of Psychiatry, University of \\ Alberta, Edmonton, Alberta, Canada. * These authors have contributed equally to this manuscript (study coordinators). ${ }^{\dagger}$ These authors have \\ contributed equally to this manuscript (principal investigators).
}

\begin{abstract}
Objective: Electroconvulsive therapy (ECT) is a well-established therapeutic intervention for major depressive disorder. Recent literature has shown that the anesthetic agent ketamine has some antidepressant properties at low doses and may be an alternative therapy for treatment-resistant major depressive disorder. We hypothesized that the use of low-dose ketamine as an anesthetic adjunct in ECT would more rapidly improve depression while maintaining hemodynamic stability than ECT with propofol alone.

Methods: Institutional ethics approval was obtained, and the use of ketamine in this study was approved by Health Canada. This is a randomized, double-blinded, placebo-controlled trial that involved ketamine administration at $0.5 \mathrm{mg} / \mathrm{kg} \mathrm{IV}$ in addition to propofol anesthesia for ECT. The primary outcome was the number of ECT treatments required to achieve a $50 \%$ reduction in the Montgomery-Asberg Depression Rating Scale (MADRS). Secondary outcomes included the number of ECT treatments required to achieve a $25 \%$ reduction in MADRS score, as well as any differences in the Clinical Global Impression Scale for Severity, hemodynamic variables, and seizure duration. Adverse events were recorded for safety assessment.

Results: A total of 45 patients completed the study. No difference was found between groups with respect to the primary or secondary outcomes. The ketamine group showed a trend towards a decreased dose of propofol required to achieve adequate anesthesia. No adverse events were reported.

Conclusion: Low-dose ketamine does not improve psychiatric outcomes in the setting of propofolbased anesthesia for ECT. Specifically, ketamine did not reduce the number of ECT sessions necessary to achieve a 50 or $25 \%$ reduction in MADRS scores. Reassuringly, the fact that no differences in hemodynamic variables or unexpected adverse events occurred suggests that low-dose ketamine may be safely used in this setting should clinical indications warrant its use.

Clinical trial registration: ClinicalTrials.gov, NCT02579642
\end{abstract}

Keywords: Depressive disorder; major/drug therapy; depressive disorder; major/therapy; ketamine/ therapeutic use; anesthesia/therapeutic use; electroconvulsive therapy/therapeutic use; ketamine/ adverse effects

\section{Introduction}

Electroconvulsive therapy (ECT) is a treatment modality used for a variety of psychiatric disorders, including major depressive disorder. It is highly effective, demonstrating an overall remission rate of up to $87 \% .{ }^{1}$ At this time, the therapeutic mechanism of ECT is largely unknown. However, theories exist relating to increases in brain concentrations of gamma-aminobutyric acid and serotonin, as well as alterations in brain-derived neurotrophic

Correspondence: Adrianna J. Woolsey, 2-150 Clinical Sciences Building, 8440112 St NW, Edmonton, Alberta, Canada T6G 2 G3. E-mail: woolsey@ualberta.ca

Submitted Dec 15 2020, accepted Feb 17 2021, Epub May 282021. factor modulation and changes in regional cerebral blood flow and metabolism. ${ }^{2,3}$ To enhance patient comfort and ensure safety, patients are typically given a short-acting general anesthetic agent, commonly propofol, as well as a short-acting paralytic. Propofol, a potent antiepileptic agent, ${ }^{4}$ has been shown to decrease seizure duration in the setting of ECT, ${ }^{5}$ which may reduce the efficacy of ECT as a depression treatment.

Ketamine is a general anesthetic agent with dissociative properties, and importantly, may prolong seizure

How to cite this article: Woolsey AJ, Nanji JA, Moreau C, Sivapalan S, Bourque SL, Ceccherini-Nelli A, et al. Low-dose ketamine does not improve the speed of recovery from depression in electroconvulsive therapy: a randomized controlled trial. Braz J Psychiatry. 2022;44:6-14. http://dx.doi.org/10.1590/1516-4446-20201705 
duration. ${ }^{6}$ Ketamine has also been used successfully as the sole anesthesia induction agent in ECT. The drawbacks of using ketamine as the sole induction agent are related to its hemodynamic and psychotomimetic effects. In addition to eliciting tachypnea in patients, ketamine causes tachycardia and hypertension at doses used to induce general anesthesia and can cause an unpleasant dissociative experience upon emergence from anesthesia. $^{7}$ At low doses, however, ketamine in isolation has been described in the literature as a successful treatment for refractory depressive disorders. ${ }^{8}$ Additionally, there is some evidence that the use of ketamine in treatmentresistant depression may also improve cognitive measures such as sustained attention and response time. ${ }^{9}$ The use of low-dose ketamine may thus improve ECT depression treatment by potentially improving seizure activity elicited by ECT or by improving depression in its own right.

Based on this information, we hypothesized that the addition of low-dose ketamine to propofol-based anesthesia would have an additive or synergistic effect with ECT in improving depression outcomes.

\section{Methods}

\section{Study overview}

This trial was conducted at the University of Alberta Hospital in Edmonton, Alberta, Canada.

\section{Study design}

This was a double-blind, placebo-controlled, randomized trial in adult patients with major depressive disorder undergoing ECT. The study was designed with an interim analysis to allow for reassessment of the intervention in an effort to enhance safety and increase the likelihood of detecting any differences. The initial 14 subjects would receive ketamine at a dose of $0.2 \mathrm{mg} / \mathrm{kg}$, which was chosen as the lowest feasible starting dose based on a literature review. An interim analysis of psychiatric endpoints would then be performed; if a trend towards a difference was detected, the study would go on to recruit an additional 14 patients, using a dose of $0.2 \mathrm{mg} / \mathrm{kg}$. However, if no difference was detected, the dose would be increased to $0.5 \mathrm{mg} / \mathrm{kg}$ (again, based on studies which found that this dose had a beneficial effect on depression while remaining within the range of a sub-anesthetic dose), and an additional 30 patients would be recruited.

\section{Subjects}

The inclusion criteria were adults between 18 and 70 years of age referred for ECT with a DSM-5 diagnosis of major depressive disorder and at least moderate to severe depression as measured by a baseline Montgomery-Asberg Depression Rating Scale (MADRS) score $>24$. The included patients were having a "first" or "new" episode of depression that had lasted not more than 3 months and who, according to a psychiatrist, required ECT treatment. The exclusion criteria were: 1) patients considered American Society of Anesthesiologists Class IV or V; patients who received any ECT treatments in the 3 months prior to enrollment; 2) a history of allergic reactions, hypersensitivity, or intolerance to anesthetics or their constituents used in the study; 3) any of the following DSM-5 diagnoses at the time of enrollment: substance or alcohol dependence (except for dependence in full remission and caffeine or nicotine dependence), abuse of opiates, amphetamines, barbiturates, cocaine, cannabis, or hallucinogens in the 4 weeks prior to enrollment, a pervasive developmental disorder or dementia; 4) a significant medical condition or concurrent medication that would contraindicate the use of ketamine or propofol or a medical condition that is untreated and requires urgent attention; 5) an unstable or inadequately treated medical illness; 6) an increased risk of laryngospasm (such as active pulmonary infection, upper respiratory infection, asthma); 7) any clinically significant deviation from the reference range in clinical laboratory test results; 8) pregnancy (or female of child-bearing age not using adequate contraception) or lactation; 9) participation in another drug trial in the 4 weeks prior to enrollment in this study; and 10) inability or refusal to provide informed consent.

\section{Randomization and blinding}

Allocation concealment was achieved using the sequentially-numbered, opaque, sealed envelope method. ${ }^{10}$ The study coordinator was responsible for creating the sealed envelopes, which were then delivered to the research pharmacy for shuffling and assignment. As a result, the study coordinator, treating psychiatrist, anesthesiologist, and patients were all blinded to treatment allocation. The study statistician was also blinded to treatment allocation when performing the analysis. The study coordinator was responsible for screening and enrolling study subjects.

\section{Study medication}

The study drug was prepared by the research pharmacy in a syringe labelled with patient identifiers, containing either ketamine (Sandoz Canada, Boucherville, QC, Canada) $0.2 \mathrm{mg} / \mathrm{kg}$ (patients 1-14) or $0.5 \mathrm{mg} / \mathrm{kg}$ (patients 15 onward) or an equivalent volume of normal saline. The syringes were then securely delivered to the ECT treatment area and immediately given to the ECT anesthesiologist. Thus, the anesthesiologist was blinded to group allocation. The anesthesiologist was instructed to administer the entire study drug syringe followed immediately by propofol (Aspen Pharmacare Canada Inc., Oakville, ON, Canada) and succinylcholine (Teligent Canada Inc., Mississauga, ON, Canada). The dosing of all agents and adjuncts was determined at the discretion of the attending anesthesiologist to achieve adequate anesthesia for the ECT treatment.

\section{Procedures}

The patients received the study drug at each of their treatments in the index course of ECT. A maximum of 
12 treatments were performed, or until the patient was transitioned to maintenance ECT therapy, whichever came first. Per protocol at our institution, patients receive ECT three times per week, on Monday, Wednesday, and Friday; therefore, a typical 12-treatment index course would span approximately four weeks. Pulse ECT was performed using a MECTA SpECTrum 5000Q device (MECTA Corp, Tualatin, OR, USA) with titrated stimulus dosing per titration protocols for unilateral ultrabrief (New York Psychiatry Institute, Columbia University, New York, NY, USA) and bilateral pulses (Henry Ford Hospital, Detroit, MI, USA). A REDCap secure online database was provided by the University of Alberta and used for data collection and management. The data are available via third-party platform REDcap (https://www. project-redcap.org/).

\section{Outcomes}

The primary outcome measure was the number of ECT sessions required to achieve a $50 \%$ reduction in the 10-item MADRS. The secondary psychiatric outcome measures were the number of ECT sessions needed to achieve a $25 \%$ reduction in the MADRS and any improvement in the 7-point Clinical Global Impression Scale for Severity (CGI-S). These scales were administered by the treating psychiatrist before the first ECT session and before every subsequent session.

Data were collected on the following non-psychiatric secondary outcomes: heart rate, blood pressure, oxygen saturation, respiratory rate, temperature, and seizure duration, which was observed both visually and by EEG. The dosing of propofol, succinylcholine, and adjunctive medications were recorded. Adverse events were collected by spontaneous report at every ECT session and during inpatient hospitalization.

\section{Statistical analysis}

Sample size calculations were based on studies examining the use of ketamine infusion for treatment-resistant major depressive disorder. Murrough et al. ${ }^{11}$ demonstrated that approximately $63 \%$ of depressed patients have some improvement after an initial infusion of ketamine. Husain et al. ${ }^{12}$ reported that $54 \%$ of their sample had responded by treatment 3 of week 1 . Based on an estimate that response (defined as a reduction in MADRS of greater than $50 \%$ ) after a single ECT treatment is rare and does not occur in more than $10 \%$ of patients, an a priori power analysis using an online tool (https://www.statstodo.com/ SSizSurvival.php) indicated that 14 subjects per group would be required for a power of $80 \%$, assuming an alpha level of 0.05 .

All analyses were completed on an intention-to-treat basis. The interim analysis was performed on primary and secondary psychiatric endpoints only; specifically, MADRS and CGI-S scores were examined using an independent samples $t$-test and Levene's test. The final analyses were performed with psychiatric data (MADRS and CGI-S outcomes) and analyzed via an independent samples $t$-test and Levene's test. Non-psychiatric outcomes, including hemodynamic and seizure data, were analyzed using an independent samples l-test. The data are presented as mean \pm standard deviation (SD). A $p$-value of $<0.05$ was considered significant.

\section{Ethics statement}

This study was approved by the health research ethics boards of the University of Alberta (study ID Pro00044771). Health Canada approval was obtained for the off-label use of ketamine. Informed verbal and written consent were obtained from all patients prior to study participation.

\section{Results}

\section{Screening and randomization}

A total of 48 patients provided informed consent for study enrollment between October 2015 and May 2019. One patient was excluded after enrollment and two patients withdrew from ECT entirely. A total of 45 patients completed the study and were included in the final analyses (Consolidated Standards of Reporting Trials [CONSORT] diagram - Figure 1). Enrollment was discontinued when the predetermined sample size was achieved.

The first 14 patients were randomized to receive either ketamine at a dose of $0.2 \mathrm{mg} / \mathrm{kg}$ or an equivalent volume of saline, with 7 receiving treatment (ketamine) and 7 receiving placebo (saline). Interim analysis revealed no difference between the groups (labeled as group $A$ and group $B$ to minimize bias); therefore, the subsequent 31 patients were randomized to receive ketamine at a dose of $0.5 \mathrm{mg} / \mathrm{kg}$ or placebo, with 16 patients ultimately receiving ketamine and 15 patients receiving placebo. The majority of patients were women (73\%). The mean age was 42.9 , and the mean baseline MADRS score was 37.1. Baseline characteristics are presented in Table 1 (pre-interim analysis) and Table 2 (post-interim analysis).

\section{Psychiatric endpoints}

At interim analysis, no significant differences in primary or secondary psychiatric outcomes were noted. In these pre-interim analysis groups, the final MADRS scores were $11.17 \pm 9.04$ and $10.71 \pm 7.93$ for the ketamine and placebo groups, respectively; and the CGI-S scores at the conclusion of ECT treatment sessions were $2.67 \pm 1.21$ and $3.14 \pm 1.35$ for the ketamine and placebo groups, respectively. In the final analyses (i.e., post-interim analysis groups), there were no significant differences in the primary or secondary outcomes, with the ketamine group achieving a $50 \%$ reduction in MADRS after $8.25 \pm 2.72$ ECT sessions, while the placebo group required an average of $7.73 \pm 2.89$ ECT sessions ( $p=0.61$; Figure $2 A)$. There was no difference in the number of ECT sessions required to achieve a $25 \%$ reduction in MADRS or in the CGI-S score between groups. The ketamine group achieved a $25 \%$ MADRS reduction in $4.25 \pm 1.52$ ECT sessions and the placebo group achieved it in $5.47 \pm 2.95 \mathrm{ECT}$ sessions $(p=0.34$; Figure 2B). The CGI-S score improved in the 


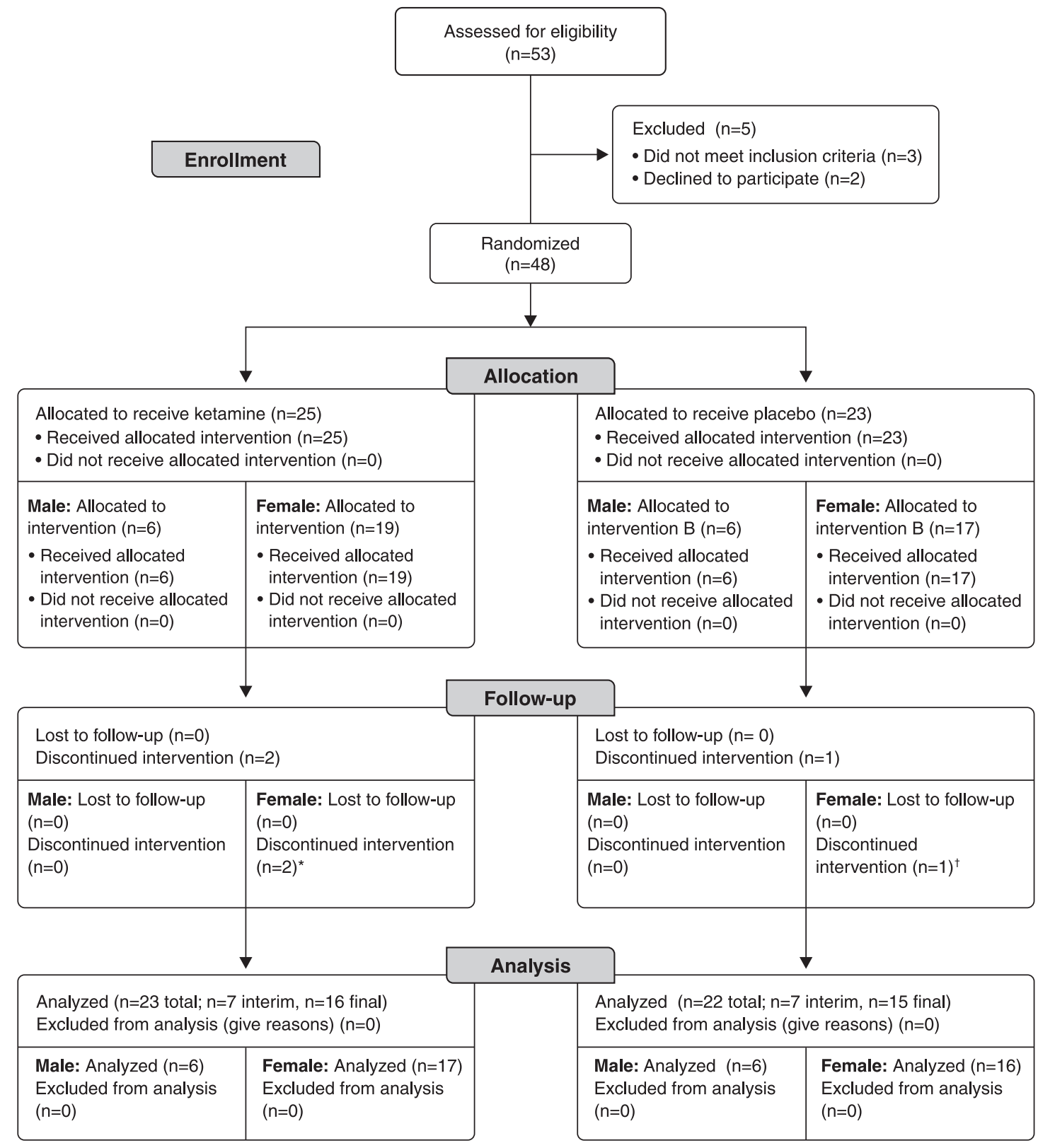

Figure 1 CONSORT diagram depicting study recruitment. Details about discontinued intervention: * 1 patient was found to be ineligible after enrollment (baseline MADRS $<23$ ); ${ }^{\dagger} 1$ patient withdrew from electroconvulsive therapy. CONSORT = Consolidated Standards of Reporting Trials; ECT = electroconvulsive therapy; MADRS = Montgomery-Asberg Depression Rating Scale.

Table 1 Baseline demographics for study participants in the pre-interim analysis

\begin{tabular}{|c|c|c|c|c|c|c|}
\hline \multirow[b]{2}{*}{ Variable } & \multicolumn{3}{|c|}{ Ketamine } & \multicolumn{3}{|c|}{ Placebo } \\
\hline & Male $(n=1)$ & Female $(n=6)$ & Combined $(n=7)$ & Male $(n=2)$ & Female $(n=5)$ & Combined $(n=7)$ \\
\hline Age (years) & 68 & $42.5 \pm 17.7$ & $46.1 \pm 18.8$ & $56.5 \pm 14.8$ & $46.0 \pm 20.8$ & $49.0 \pm 18.7$ \\
\hline Baseline MADRS & $\begin{array}{l}32.3 \\
42\end{array}$ & $\begin{array}{l}21.8 \pm 3.8 \\
38.2 \pm 6.2\end{array}$ & $\begin{array}{l}23.3 \pm 5.3 \\
38.8 \pm 5.8\end{array}$ & $\begin{array}{l}32.3 \pm 8.9 \\
40.0 \pm 11.3\end{array}$ & $\begin{array}{l}28.9 \pm 9.4 \\
36.6 \pm 8.7\end{array}$ & $\begin{array}{l}29.8 \pm 8.6 \\
37.8 \pm 8.6\end{array}$ \\
\hline Baseline CGI-S & 5 & $5.2 \pm 0.4$ & $5.2 \pm 0.4$ & $5.0 \pm 1.4$ & $5.0 \pm 0.7$ & $5.0 \pm 0.8$ \\
\hline Duration of depressive episode (months) & 2 & $2.3 \pm 0.9$ & $2.2 \pm 0.8$ & $3.0 \pm 0.0$ & $2.7 \pm 0.6$ & $2.8 \pm 0.4$ \\
\hline
\end{tabular}

$\mathrm{BMI}=$ body mass index; CGI-S = Clinical Global Impression Scale for Severity; MADRS = Montgomery-Asberg Depression Rating Scale. 
Table 2 Baseline demographics for study participants in the post-interim analysis

\begin{tabular}{lccccccc}
\hline & \multicolumn{3}{c}{ Ketamine } & & \multicolumn{3}{c}{ Placebo } \\
\cline { 2 - 3 } Sex & Male $(\mathrm{n}=5)$ & Female $(\mathrm{n}=11)$ & Combined $(\mathrm{n}=16)$ & & Male $(\mathrm{n}=4)$ & Female $(\mathrm{n}=11)$ & Combined $(\mathrm{n}=15)$ \\
\hline Age (years) & $29.4 \pm 15.2$ & $40.4 \pm 14.2$ & $36.9 \pm 15.0$ & & $45.0 \pm 11.4$ & $45.0 \pm 12.0$ & $45.0 \pm 11.5$ \\
BMI (kg/m $\left.{ }^{2}\right)$ & $28.0 \pm 8.3$ & $31.0 \pm 8.8$ & $30.0 \pm 8.5$ & & $30.6 \pm 5.3$ & $30.2 \pm 10.6$ & $30.3 \pm 9.3$ \\
Baseline MADRS & $32.8 \pm 6.5$ & $39.5 \pm 8.2$ & $37.4 \pm 8.2$ & & $34.0 \pm 4.2$ & $36.5 \pm 7.3$ & $35.9 \pm 6.5$ \\
Baseline CGI-S & $4.2 \pm 0.4$ & $4.7 \pm 0.8$ & $4.6 \pm 0.7$ & & $3.5 \pm 2.4$ & $4.1 \pm 1.4$ & $3.9 \pm 1.7$ \\
Duration of depressive & $1.8 \pm 0.4$ & $2.3 \pm 0.8$ & $2.1 \pm 0.7$ & & $1.8 \pm 1.0$ & $2.0 \pm 0.4$ & \\
episode (months) & & & & & &
\end{tabular}

$\mathrm{BMI}=$ body mass index; CGI-S = Clinical Global Impression Scale for Severity; MADRS = Montgomery-Asberg Depression Rating Scale.

A MADRS reduction by $50 \%$

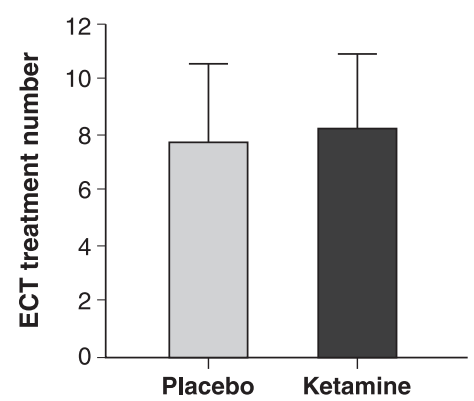

B

MADRS reduction by $25 \%$

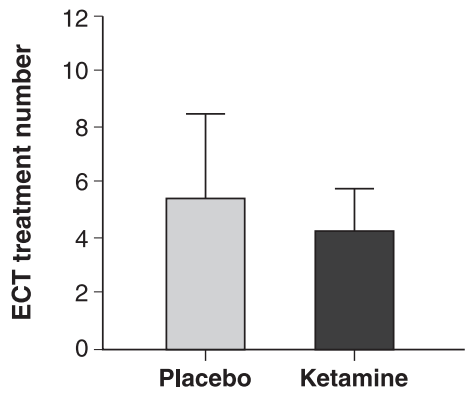

C

CGI-S

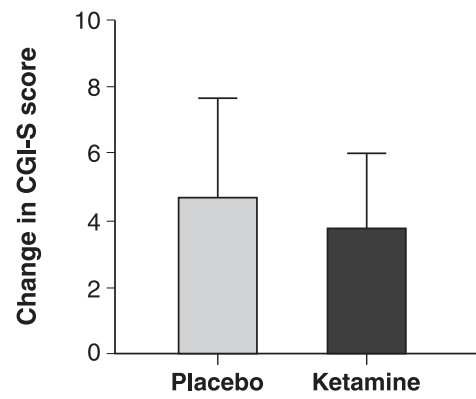

Figure 2 Reduction of MADRS and CGI-S scores following ECT with or without ketamine. The cumulative data (mean values \pm SD) showed no significant difference in the number of ECT sessions required to reduce MADRS scores by (A) 50 or (B) $25 \%$ between the ketamine and placebo groups. C) The CGI-S score reduction did not differ significantly between the groups. $\mathrm{n}=16$ for the ketamine group and $\mathrm{n}=15$ for the placebo group. CGI-S = Clinical Global Impression Scale for Severity; ECT = electroconvulsive therapy; MADRS = Montgomery-Asberg Depression Rating Scale; SD = standard deviation.

A

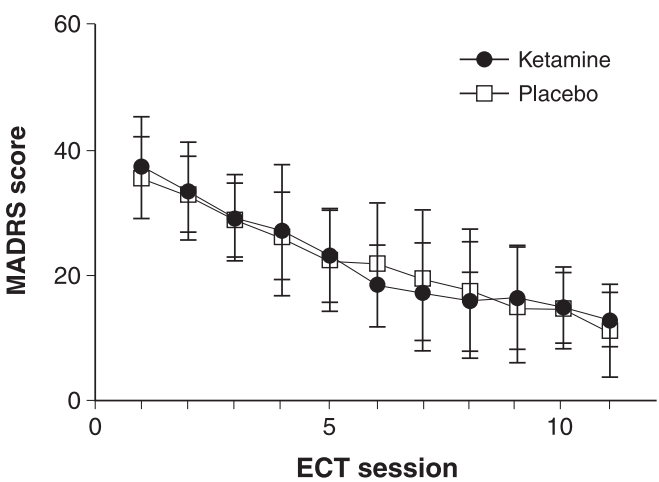

B

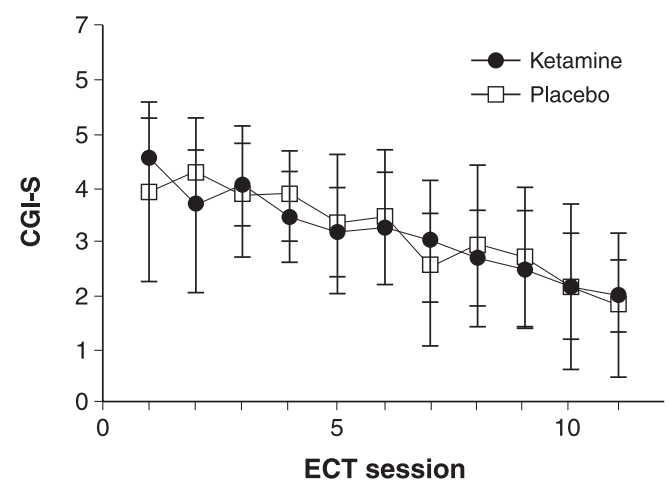

Figure 3 Reduction of MADRS and CGI-S scores over the duration of ECT sessions. Cumulative data (mean values \pm SD) showing the reduction in (A) MADRS scores and (B) CGI-S scores during ECT in the ketamine and placebo groups. $n=16$ for the ketamine group and $n=15$ for the placebo group. CGI-S = Clinical Global Impression Scale for Severity; ECT = electroconvulsive therapy; MADRS = Montgomery-Asberg Depression Rating Scale; SD = standard deviation.

ketamine group by $3.75 \pm 2.30$ and by $4.73 \pm 2.98$ the placebo group ( $p=0.26$; Figure $2 \mathrm{C}$ ). The final MADRS scores at the conclusion of the ECT sessions were $13.11 \pm 4.83$ for the ketamine group and $10.83 \pm 6.86$ for the placebo group. The final CGI-S scores at the conclusion of ECT sessions were 1.67 \pm 1.23 for the ketamine group and $1.58 \pm 1.17$ for the placebo group. Figure 3 shows the change in mean MADRS and CGI-S scores over the duration of ECT treatment.

\section{Non-psychiatric endpoints}

The maximum heart rate was significantly higher in the ketamine group than the placebo group (98.64 \pm 8.06 vs. $90.32 \pm 12.77$ beats per minute [bpm]; $p=0.037$; Figure $4 \mathrm{~A}$ ). The mean arterial pressure (MAP) did not differ significantly between the ketamine and placebo groups (112.64 \pm 11.75 vs. $110.70 \pm 7.98 \mathrm{mmHg}$; $p=0.60$; Figure 4B). However, when the maximum heart 

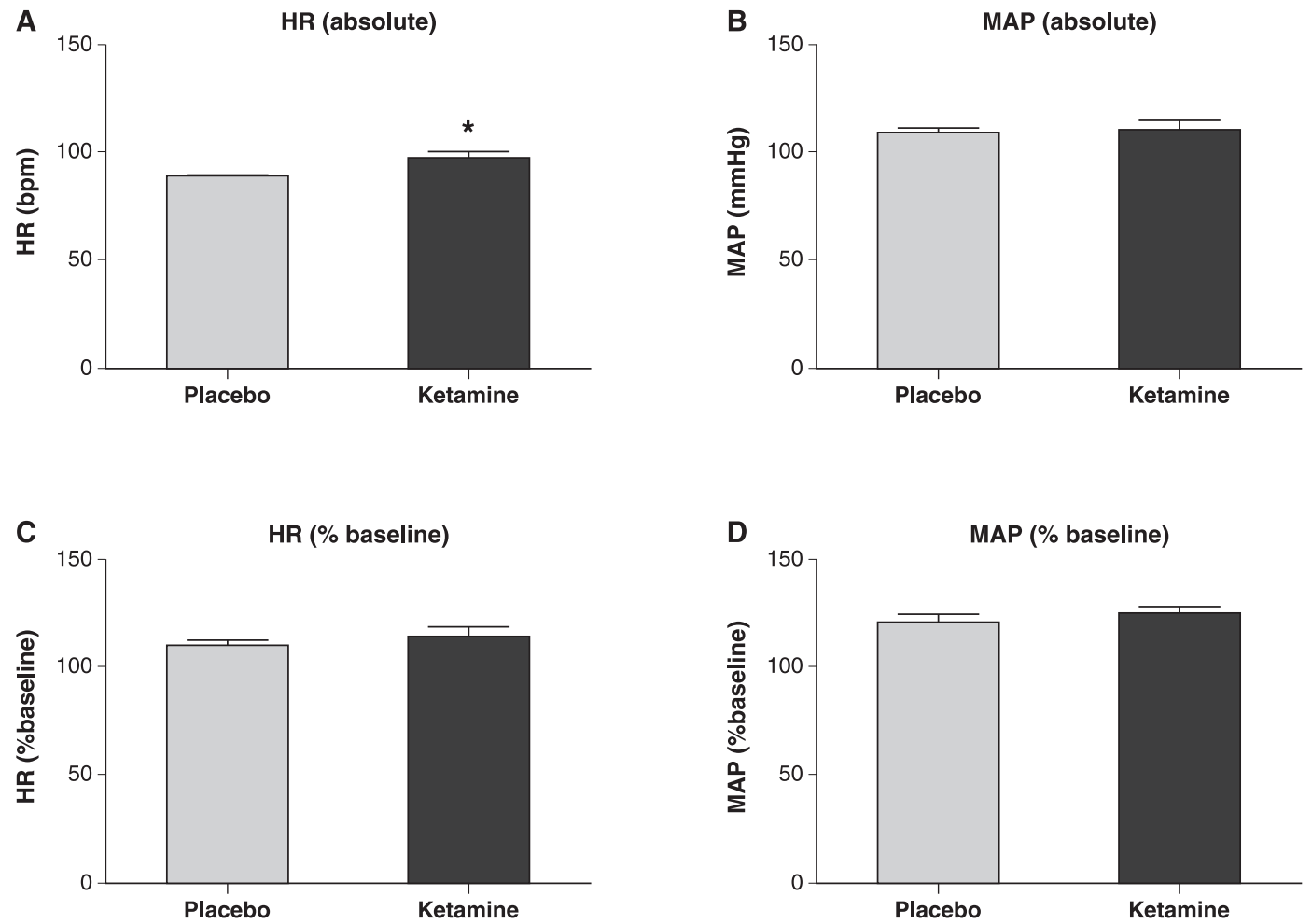

Figure 4 Hemodynamic alterations in the ketamine and placebo groups during ECT. A) The cumulative data (mean values \pm $\mathrm{SD})$ showed that maximum HR increased significantly in the ketamine group compared to placebo group $\left({ }^{*} p<0.05\right)$. B). The cumulative data (mean values \pm SD) showed that MAP did not significantly differ between the ketamine and placebo groups. When normalized to each patient's baseline hemodynamic parameters, the cumulative data (mean values \pm SD) revealed no significant difference in (C) maximum HR or (D) maximum mean arterial pressure. $n=16$ for the ketamine group and $n=15$ for the placebo group. $\mathrm{ECT}=$ electroconvulsive therapy; $\mathrm{HR}=$ heart rate; $\mathrm{MAP}=$ mean arterial pressure; $\mathrm{SD}=\mathrm{standard}$ deviation.

rate and MAP were normalized to the patient's baseline pre-ECT values, they did not differ significantly between the groups (\% heart rate increase in ketamine vs. placebo was $115.93 \pm 14.59$ vs. $111.78 \pm 13.74$, $\mathrm{p}=0.42 ; \%$ MAP increase in ketamine vs. placebo was $125.47 \pm 10.22$ vs. $122.65 \pm 9.67, p=0.44$; Figures 4C and 4D).

Propofol dosing was examined for both the interim (ketamine $0.2 \mathrm{mg} / \mathrm{kg}$ ) and final (ketamine $0.5 \mathrm{mg} / \mathrm{kg}$ ) analyses. No difference in total dose was found in the interim analysis, with the ketamine group receiving propofol at $1.21 \pm 0.47 \mathrm{mg} / \mathrm{kg}$ and the placebo group receiving propofol at $1.25 \pm 0.24 \mathrm{mg} / \mathrm{kg} \quad(p=0.85$; Figure 5A). In the final analysis, a trend towards a lower dose of propofol was observed in the ketamine group, which received $1.10 \pm 0.31 \mathrm{mg} / \mathrm{kg}$ vs. $1.36 \pm 0.39 \mathrm{mg} / \mathrm{kg}$ in the placebo group ( $p=0.053$; Figure $5 B$ ). Seizure duration did not significantly differ between the group (35.74 \pm 11.82 vs. $35.09 \pm 6.97 \mathrm{sec}, \mathrm{p}=0.86$; Figure $5 \mathrm{C}$ ). Finally, the mean maximum post-ECT respiratory rate for the ketamine group was $23.71 \pm 1.90$ vs. $23.20 \pm 2.22$ for the placebo group ( $p=0.50$; Figure 5D).

\section{Safety and tolerability}

No unexpected adverse events were reported.

\section{Discussion}

In this randomized controlled trial, adding low-dose ketamine to a standard propofol-based anesthetic regimen for ECT did not result in better depression scores than placebo. Although low-dose ketamine may reduce the anesthetic requirements for propofol during ECT treatment, there were no differences in hemodynamic profile or seizure duration with low-dose ketamine compared to placebo.

The use of ketamine in depression treatment has garnered much interest in the last decade, and the literature on the subject has increased considerably. Numerous randomized controlled trials ${ }^{13-23}$ and several meta-analyses ${ }^{8,24-26}$ have been conducted. Although a variety of protocols involving ketamine has been tested, the results have been equivocal, with inconsistent evidence of improvement in psychiatric outcomes and some evidence of potential for increased harm. For example, a meta-analysis by McGirr et al. ${ }^{8}$ compared standard anesthetic agents for ECT vs. adjunct ketamine and found no significant improvement in symptomatology, whereas Zheng et al. ${ }^{25}$ found increased early improvement in patients who received ketamine. $\mathrm{Li}$ et al. ${ }^{26}$ found sustained increased improvement in the ketamine group, although they also found a significantly greater risk of delirium and confusion, hallucination, 

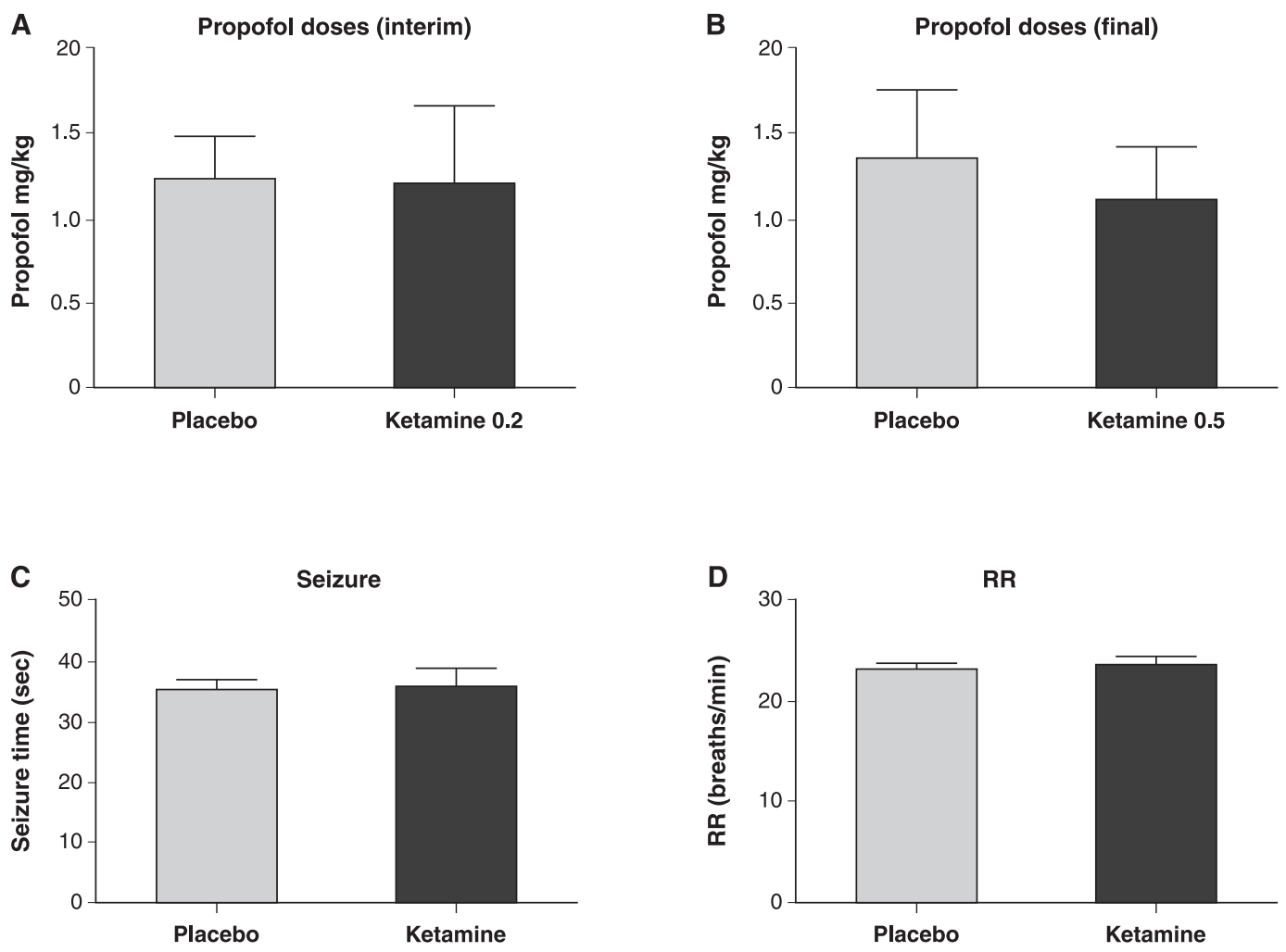

Figure 5 Differences in propofol dosing, seizure duration, and maximum respiratory rate between the ketamine and placebo groups during electroconvulsive therapy. A) Cumulative data (mean values \pm SD) demonstrating that propofol dosing did not differ significantly in the presence of ketamine $0.2 \mathrm{mg} / \mathrm{kg}$. B) Cumulative data (mean values \pm SD) demonstrating a non-significant decrease in propofol requirements in the presence of ketamine $0.5 \mathrm{mg} / \mathrm{kg}(\mathrm{p}=0.053)$. The cumulative data (mean values $\pm S D$ ) also revealed no significant difference in $(C)$ seizure duration or $(D)$ maximum respiratory rate between the ketamine and placebo groups. $n=16$ for the ketamine group and $n=15$ for the placebo group. $R R=$ respiratory rate; $\mathrm{SD}=$ standard deviation

headache, hypertension, and tachycardia in patients who received ketamine compared to other anesthetics. Our study differs from previous trials in that we started with the lowest dose of ketamine reported to improve depression $(0.2 \mathrm{mg} / \mathrm{kg})$ and then performed an interim analysis, at which point we determined that an increased dose was indicated. This allowed us to "titrate" the study drug to ensure safety and increase the probability that potential differences, if any, would be identified early. Our design is also unique in that the study investigators, psychiatrists, and the treating anesthesiologist were all blinded to the allocation. Furthermore, we allowed the anesthesiologist to select the propofol dose. Despite being blinded, the anesthesiologists were able to "titrate" the effect of propofol over multiple ECT sessions, thus enhancing the authenticity of the clinical scenario and avoiding excessive anesthesia. Additionally, most studies have administered the study drug to patients for a maximum of 6 to 8 treatments, whereas our study included up to 12 treatments, which increased the chance of detecting any true difference in outcomes.

Although our results did not suggest that psychiatric outcomes improve with ketamine as an adjunct to ECT, ketamine was well tolerated at the doses we used, with no unexpected adverse events reported. One finding that has emerged in other studies with designs similar to ours $^{23}$ was an early increased improvement in the ketamine groups that is attenuated with subsequent ECT treatments. This might indicate that there is a ceiling effect to ECT: the patients achieved maximal recovery after an index course of therapy plus ketamine, which although possibly useful on its own in the short term, does not alter long-term ECT outcomes. Perhaps ketamine could be employed after an index course of ECT as a form of maintenance therapy. This is an area that remains to be explored.

Another reason that ketamine provided no additional benefits to ECT could be that ketamine and ECT share similar antidepressant mechanisms. ECT is believed to work through various mechanisms, including changes in cerebral blood flow and regional metabolism ${ }^{2}$ and breakdown of the blood brain barrier related to periods of hypertension. ${ }^{3}$ ECT may also increase regional concentrations of brain-derived neurotrophic factor, a protein that may be deficient in some depression patients. ${ }^{27}$ Ketamine is known to increase regional cerebral blood flow $^{28}$ and the cerebral metabolic rate in a similar manner. It may elicit a hypertensive response similar to ECT, and it also causes upregulation of brain-derived neurotrophic factor. $^{2} \mathrm{~N}$-methyl-D-aspartate receptor 
antagonism, although purported to have antidepressant action, may not be relevant in this situation. Indeed, other N-methyl-D- aspartate receptor antagonists do not seem to have the same therapeutic effects when tested. $^{29}$ Perhaps adding ketamine to ECT is not beneficial because there is no true opportunity for additive or synergistic effects, which otherwise may be seen with differing mechanisms of action between two different treatment modalities.

A third explanation could be that the benefits of ketamine on its own in an outpatient setting are specifically related to the dissociative experience of receiving ketamine rather than any pharmacodynamic effects. This effect would be similar to the dissociative experience of taking hallucinogenic drugs such as ayahuasca, which has been shown to improve depression. ${ }^{30}$ If this were the case, it might explain why ketamine has little benefit when administered as part of a general anesthetic technique, since its dissociative effects may be offset by general anesthesia.

Given that the anesthesiologist was unaware of the treatment allocation but could still control the dose of propofol, the first treatment was based on typical clinical propofol dosing for ECT. A potential pitfall here is that the blinding of the anesthesiologist may have led to a relative propofol overdose in early treatments, possibly affecting the results. At our center, a different anesthesiologist administers ECT each day of the week. At the end of each treatment, the anesthesiologist documents the dosage and any recommendations about dose modification for subsequent treatments. A trend towards a decreased dose of propofol in the ketamine group would be expected (and was confirmed in our results), which indicates that even with blinding, the propofol doses were, in essence, appropriately titrated to effect. In light of this information, it is interesting that we did not find a difference in seizure duration or hemodynamic effects in these patients. Based on the results of our study, we would caution against the routine use of low-dose ketamine as an adjunct to propofol-based ECT for patients with insufficient seizure duration since low-dose ketamine may reduce propofol requirements and thus theoretically increase seizure duration. Given that our study was not designed to directly assess this, and considering the previously mentioned concern about relative propofol overdose due to blinding, it is still possible that this approach might be valid in certain situations. However, it is reassuring that our results suggest that low-dose ketamine is a safe addition to the anesthetic regimen for ECT. Fears of hemodynamic derangement should not be a barrier to the use of ketamine in this regard.

One limitation of our study is the small sample size. However, given that there were no trends toward differences between the groups regarding the psychiatric endpoints, meaningful information can still be conveyed in this regard. In addition, due to the sample size, a complete analysis of the data at different time points in non-psychiatric secondary outcomes was not possible. Another limitation is that post-treatment cognitive testing was not performed, thus any subtle cognitive effects due to ketamine could not be determined. Finally, since we did not formally assess time spent in the post-anesthesia care unit, we cannot comment on whether low-dose ketamine prolongs recovery time after ECT.

In conclusion, low-dose ketamine in combination with ECT did not improve depression compared to placebo. Specifically, ketamine did not reduce the number of ECT sessions necessary to achieve a 50 or $25 \%$ reduction in MADRS scores. Low-dose ketamine also did not affect hemodynamic parameters during ECT treatment, and seizure duration did not differ from placebo, despite potentially decreasing the dose of propofol required to induce satisfactory anesthesia for ECT. Therefore, lowdose ketamine may be considered for use in certain clinical contexts for ECT treatment, although it should not be used solely for improving depression in the setting of ECT.

\section{Acknowledgements}

This project was funded by the division of Addiction and Mental Health, Edmonton Zone, Alberta Health Services.

We would like to acknowledge the staff of the electroconvulsive therapy suite at the University of Alberta Hospital.

\section{Disclosure}

CM participated in the Regional Canadian Esketamine Advisory Board Meeting (AB-SK-MB, Feb 13 2019), which provided a clinical review of Esketamine, examined evidence for its use, and reviewed safety protocols. The other authors report no conflicts of interest.

\section{References}

1 Petrides G, Fink M, Husain MM, Knapp RG, Rush AJ, Mueller M, et al. ECT remission rates in psychotic versus nonpsychotic depressed patients: a report from CORE. J ECT. 2001;17:244-53.

2 Takano H, Motohashi N, Uema T, Ogawa K, Ohnishi T, Nishikawa M, et al. Differences in cerebral blood flow between missed and generalized seizures with electroconvulsive therapy: a positron emission tomographic study. Epilepsy Res. 2011;97:225-8.

3 Andrade C, Bolwig TG. Electroconvulsive therapy, hypertensive surge, blood-brain barrier breach, and amnesia: exploring the evidence for a connection. J ECT. 2014;30:160-4.

4 Zhang Q, Yu Y, Lu Y, Yue H. Systematic review and meta-analysis of propofol versus barbiturates for controlling refractory status epilepticus. BMC Neurol. 2019;19:55.

5 Jarineshin H, Kashani S, Fekrat F, Vatankhah M, Golmirzaei J, Alimolaee E, et al. Seizure duration and hemodynamic state during electroconvulsive therapy: sodium thiopental versus propofol. Glob J Health Sci. 2015;8:126-31.

6 Jagtiani A, Khurana $H$, Malhotra N. Comparison of efficacy of ketamine versus thiopentone-assisted modified electroconvulsive therapy in major depression. Indian J Psychiatry. 2019;61:258-64.

7 Peltoniemi MA, Hagelberg NM, Olkkola KT, Saari TI. Ketamine: a review of clinical pharmacokinetics and pharmacodynamics in anesthesia and pain therapy. Clin Pharmacokinet. 2016;55:1059-77.

8 McGirr A, Berlim MT, Bond DJ, Fleck MP, Yatham LN, Lam RW. A systematic review and meta-analysis of randomized, double-blind, placebo-controlled trials of ketamine in the rapid treatment of major depressive episodes. Psychol Med. 2015;45:693-704.

9 Chen MH, Li CT, Lin WC, Hong CJ, Tu PC, Bai TM, et al. Cognitive function of patients with treatment-resistant depression after a single low dose of ketamine infusion. J Affect Disord. 2018;241:1-7.

10 Doig GS, Simpson F. Randomization and allocation concealment: a practical guide for researchers. J Crit Care. 2005;20:187-91; discussion 191-3. 
11 Murrough JW, losifescu DV, Chang LC, Al Jurdi RK, Green CE, Perez AM, et al. Antidepressant efficacy of ketamine in treatmentresistant major depression: a two-site randomized controlled trial Am J Psychiatry. 2013;170:1134-42.

12 Husain MM, Rush AJ, Fink M, Knapp R, Petrides G, Rummans T, et al. Speed of response and remission in major depressive disorder with acute electroconvulsive therapy (ECT): a Consortium for Research in ECT (CORE) report. J Clin Psychiatry. 2004;65:485-91.

13 Abdallah C, Fasula M, Kelmendi B, Sanacora G, Ostroff R. Rapid antidepressant effect of ketamine in the electroconvulsive therapy setting. J ECT. 2012;28:157-61.

14 Carspecken CW, Borisovskaya A, Lan ST, Heller K, Buchholz J, Ruskin D, et al. Ketamine anesthesia does not improve depression scores in electroconvulsive therapy: a randomized clinical trial. J Neurosurg Anesthesiol. 2018;30:305-13.

15 Fernie G, Currie J, Perrin JS, Stewart CA, Anderson V, Bennett DM, et al. Ketamine as the anaesthetic for electroconvulsive therapy: the KANECT randomised controlled trial. Br J Psychiatry. 2017;210: 422-8.

16 Gamble JJ, Bi H, Bowen R, Weisgerber G, Sanjanwala R, Prasad R, et al. Ketamine-based anesthesia improves electroconvulsive therapy outcomes: a randomized-controlled study. Can J Anaesth. 2018;65: 636-46.

17 Loo CK, Katalinic N, Garfield JB, Sainsbury K, Hadzi-Pavlovic D, Mac-Pherson R. Neuropsychological and mood effects of ketamine in electroconvulsive therapy: a randomised controlled trial. J Affect Disord. 2012;142:233-40.

18 Ray-Griffith SL, Eads LA, Han X, Golden K, Stowe ZN. A randomized pilot study comparing ketamine and methohexital anesthesia for electroconvulsive therapy in patients with depression. J ECT. 2017;33: 268-71.

19 Salehi B, Mohammadbeigi A, Kamali AR, Taheri-Nejad MR, Moshiri I. Impact comparison of ketamine and sodium thiopental on anesthesia during electroconvulsive therapy in major depression patients with drug-resistant; a double-blind randomized clinical trial. Ann Card Anaesth. 2015;18:486-90.

20 Alizadeh NS, Maroufi A, Nasseri K, Najafabadi SH, Taghiabad AM, Gharibi F, et al. Antidepressant effect of combined ketamine and electroconvulsive therapy on patients with major depressive disorder: a randomized trial. Iran J Psychiatry Behav Sci. 2015;9:e1578.
21 Yoosefi A, Sepehri AS, Kargar M, Akhondzadeh S, Sadeghi M, Rafei $A$, et al. Comparing effects of ketamine and thiopental administration during electroconvulsive therapy in patients with major depressive disorder: a randomized, double-blind study. J ECT. 2014;30:15-21.

22 Zhang M, Rosenheck R, Lin X, Li Q, Zhou Y, Xiao Y, et al. A randomized clinical trial of adjunctive ketamine anesthesia in electroconvulsive therapy for depression. J Affect Disord. 2018;227:372-8.

23 Zhong $\mathrm{X}, \mathrm{He} \mathrm{H}$, Zhang $\mathrm{C}$, Wang Z, Jiang M, Li Q, et al. Mood and neuropsychological effects of different doses of ketamine in electroconvulsive therapy for treatment-resistant depression. J Affect Disord. 2016;201:124-30.

24 Fond G, Bennabi D, Haffen E, Brunel L, Micoulaud-Franchi JA, Loundou A, et al. A Bayesian framework systematic review and meta-analysis of anesthetic agents effectiveness/tolerability profile in electroconvulsive therapy for major depression. Sci Rep. 2016;6:19847.

25 Zheng W, Li XH, Zhu XM, Cai DB, Yang XH, Ungvari GS, et al. Adjunctive ketamine and electroconvulsive therapy for major depressive disorder: a meta-analysis of randomized controlled trials. $\mathrm{J}$ Affect Disord. 2019;250:123-31.

26 Li DJ, Wang FC, Chu CS, Chen TY, Tang CH, Yang WC, et al. Significant treatment effect of add-On ketamine anesthesia in electroconvulsive therapy in depressive patients: a meta-analysis. Eur Neuropsychopharmacol. 2017;27:29-41.

27 Lee BH, Kim YK. The roles of BDNF in the pathophysiology of major depression and in antidepressant treatment. Psychiatry Investig. 2010;7:231-5.

28 Holcomb HH, Lahti AC, Medoff DR, Weiler M, Tamminga CA. Sequential regional cerebral blood flow brain scans using PET with $\mathrm{H} 2150$ demonstrate ketamine actions in CNS dynamically. Neuropsychopharmacology. 2001;25:165-72.

29 Newport DJ, Carpenter LL, McDonald WM, Potash JB, Tohen M Nemeroff CB. Ketamine and other NMDA antagonists: early clinical trials and possible mechanisms in depression. Am J Psychiatry. 2015;172:950-66.

30 Palhano-Fontes F, Barreto D, Onias $\mathrm{H}$, Andrade KC, Novaes MM, Pessoa JA, et al. Rapid antidepressant effects of the psychedelic ayahuasca in treatment-resistant depression: a randomized placebocontrolled trial. Psychol Med. 2019;49:655-63. 